\title{
Beyond Lingual Orthodontics Vol. 1: Lingual Biomechanics
}

Author: Roberto Lapenta

Publisher: Quintessence Publishing

Language: English

ISBN: 978-84-89873-64-3

Edition: 1/e

Publish Year: 2016

Pages: 604, illustrated

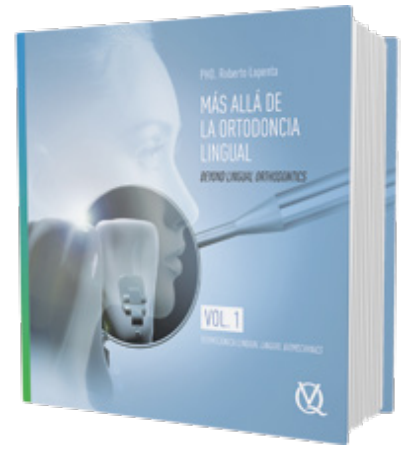

Price: $180.00 €$

An orthodontist in daily practice is very frequently required by patients to use lingual orthodontic treatment for aesthetic reasons. Dr Roberto Lapenta's book entitled "Beyond Lingual Orthodontics. Vol. 1: Lingual Biomechanics" gathers his experience of lingual therapy including mistakes and achievements, successes and failures and presents useful procedures for lingual orthodontics. The book has ten chapters and is written in Spanish and English (two-column format), and each chapter has a QR code that links to demonstration videos online.

The first two chapters tackle procedures and arguments that help us develop customized protocols for each case and patient. He believes that it is very important to understand the specific biomechanics of orthodontics and in the next two chapters he shows all lingual brackets used and different methods for positioning. In chapters five and six reveals the fundamental issue of bonding considering that $30 \%$ of the treatment success is based on the bonding of brackets including their positioning, the individualization of prescription and the optimization of bonding. Facilitating many treatments is due to the use microimplants and anchorage which are explained in chapters seven and eight. The last two chapters analyze the friction and the resolution of dental crowding with lingual orthodontics.

Dr Roberto Lapenta and his collaborators have been able to provide readers with a well-structured and photographic documented book that is a step-by-step guide for improving lingual orthodontic treatment.

DOI: 10.25241/stomaeduj.2017.4(4).bookreview.4 